\title{
Medicalización del manejo de la pandemia en Colombia: la salud pública vuelve a perder
}

\author{
Medicalization of pandemic management in Colombia: \\ public health loses again
}

\begin{abstract}
Alvaro J. Idrovo ${ }^{1}$ (i) 旅
Forma de citar: Idrovo AJ. Medicalización del manejo de la pandemia en Colombia: la salud pública vuelve a perder. Salud UIS. 2022; 54: e22001. doi: https://doi.org/10.18273/saluduis.54.e:22001@) (1)

idrovoaj@uis.edu.co

Hace ya casi medio siglo empezó a hablarse de medicalización, siendo pioneros los trabajos de Thomas Szasz, R. D. Laing y Michael Foucault ${ }^{1}$. Pese a ello, la medicalización no tiene una única definición, pero sí tiene muy claro que el control social es un elemento fundamental en el concepto. En general se puede decir que cuando se habla de medicalización se hace referencia al control social que se hace al usar el enfoque médico sobre la sociedad que, en casos extremos, puede ocasionar efectos iatrogénicos ${ }^{2}$, o expresar los problemas sociales en términos médicos ${ }^{3}$. De esta manera se hacen diagnósticos y se brindan tratamientos, por ejemplo, para problemas sociales tan diversos como la educación, la criminalidad y la economía, fuera del ámbito de la medicina y "naturalizando" (reduciendo a "leyes naturales") las sociedades.
\end{abstract}

La pandemia de COVID-19, como cualquier otra epidemia, es un problema sanitario poblacional; pese a que esta es una verdad de Perogrullo, para muchos es la sumatoria de muchas personas enfermas de COVID-19 al mismo tiempo, lo cual estrictamente no es lo mismo. Geoffrey Rose en su clásico libro "The Strategy of Preventive Medicine" (La estrategia de la Medicina Preventiva) es contundente en identificar claras diferencias al manejar individuos enfermos y poblaciones enfermas ${ }^{5}$. En el primer caso se asume, implícita o explícitamente, una aproximación basada en el "alto riesgo", mientras que en la aproximación poblacional se tiene un enfoque grupal o de colectivo ${ }^{4,5}$.

Esta diferencia no es trivial, pues se tiene evidencia de que las intervenciones o acciones poblacionales tienen mayor efectividad que las intervenciones médicas individuales. El pionero en estos trabajos fue Thomas McKeown, quien en su famoso libro "Role of medicine: dream, mirage, or nemesis?" (El rol de la medicina: sueño, milagro o némesis) publicado en 1976, indicaba que cerca de 23 años se habían añadido a la vida de una persona con intervenciones de salud pública (principalmente higiénico-sanitarias), mientras las intervenciones médicas apenas lograban incrementar cerca de 3 años la vida. Posteriores estudios han hecho estimaciones del rol de la medicina en la salud poblacional con variados hallazgos; por ejemplo, Bunker et al. usando datos de Inglaterra y Gales concluye que durante la primera mitad del siglo XX los mayores logros en salud se dieron por medidas de salud pública, y que los servicios médicos han aumentado un poco sus logros gracias a los nuevos desarrollos científicos y tecnológicos, pero sin llegar a tener nunca la importancia de las intervenciones de salud pública ${ }^{7}$. Por su parte, Kaplan y Milstein compararon cuatro métodos para evaluar el impacto de los servicios de salud con diversas fuentes de datos de Estados Unidos de América, y concluyeron que la atención médica es responsable solo de 5 a $15 \%$ de la disminución en la muerte prematura, y el resto se asocia con "circunstancias sociales" y "patrones de conducta", donde se ubican precisamente las intervenciones de salud pública. Un estudio reciente con datos de Estados Unidos

1. Universidad Industrial de Santander. Bucaramanga, Colombia 
de América entre 1990 y 2015 indica que la esperanza de vida aumentó en ese periodo 2,9 años, siendo $44 \%$ asociado a intervenciones de salud pública, $35 \%$ a medicamentos, $13 \%$ a la atención médica, y el resto a factores desconocidos 9 .

Como en todos los países, Colombia ha incorporado diversos elementos en el manejo de la pandemia, tanto de la aproximación poblacional como del enfoque de "alto riesgo". En el primer grupo se encuentran gran parte de las llamadas intervenciones no farmacológicas, como el distanciamiento social con expresiones como las cuarentenas, los aislamientos de infectados, las restricciones de movilidad, y el conjunto de acciones enmarcadas en lo que es la vigilancia en salud pública; acciones como el uso de tapabocas y el lavado de manos, son medidas no farmacológicas de carácter individual con repercusiones en la transmisión, pero con beneficios más individuales que poblacionales. Todas estas medidas fueron las que fundamentaron la respuesta durante los primeros meses de la pandemia, que en conjunto buscaron ganar tiempo para mejorar la capacidad de respuesta en los servicios de salud (mayor número de camas hospitalarias y de unidades de cuidado intensivo, por ejemplo), y mejorar la vigilancia en salud pública con incremento de personal realizando actividades de epidemiología de campo, aumentando la capacidad diagnóstica en los laboratorios y la vigilancia genómica.

Pese a los grandes esfuerzos por tener una buena vigilancia en salud pública, la realidad nos mostró que no fue realizada de manera adecuada en todos los territorios ${ }^{10}$, por el variado interés de los tomadores de decisión, y las diversas formas de gestión en los territorios ${ }^{11}$. No fue suficiente tener excelentes documentos técnicos señalando qué hacer, ni siquiera con el programa de Prueba, Rastreo y Aislamiento Selectivo Sostenible (PRASS), cuyo diseño es impecable. La evidencia de ello se resume en dos publicaciones de académicos y funcionarios del Ministerio de Salud y Protección Social; en uno se muestra la efectividad del PRASS para disminuir las muertes ${ }^{12}$, y en el segundo se muestra la costo-efectividad para el sistema de salud, cuando se realizan bien los aislamientos y diagnósticos para disminuir las muertes ${ }^{13}$. Lastimosamente, en la práctica el buen desempeño fue la excepción y no la regla. Estas limitaciones no resultan inesperadas si se tiene en cuenta que el sistema de salud colombiano, por su enfoque en priorizar la atención médica individualizada y en centros hospitalarios, ha limitado las acciones e intervenciones poblacionales propias de la salud pública.

Los determinantes de una vigilancia en salud pública con capacidad limitada sin duda son muy variados, pero no se debe desconocer la complejidad de la realidad colombiana, llena de desigualdades e inequidades profundas, más difíciles de abordar en medio de una crisis sanitaria, que llevó a un fuerte descontento social con políticas económicas, educativas, laborales y, obviamente, de salud $\mathrm{d}^{14,15}$. Este cambio en la percepción del riesgo por parte de la ciudadanía lastimosamente no se reflejó en un cambio en la estrategia de vigilancia en salud pública, quizá porque ya habían aparecido en la escena las vacunas, que cambiaron profundamente la forma de entender el manejo de la pandemia; el énfasis en las políticas de vacunación, fundamentadas en un plan nacional muy bien diseñado, rezagó la importancia de la vigilancia en salud pública que se volcó principalmente a los resultados de la mejorada, pero insuficiente, vigilancia genómica que permitió hacer seguimiento a las variantes del virus.

La vacunación contra COVID-19 es muy importante para la salud pública, porque históricamente hay hechos que muestran que las vacunaciones contra otras enfermedades pueden tener impactos enormes, tanto en la protección individual al que se vacune, como poblacional al reducir los efectos severos y la transmisión viral. Sin embargo, el proceso de vacunar a la población debería tener unos imperativos éticos y una lógica epidemiológica para tener grandes beneficios, pues no es solamente aumentar el número de vacunados. El plan nacional de vacunación original tenía plasmado todos estos elementos, pero con la mayor disponibilidad de vacunas y, luego, la llegada de la variante ómicron se hicieron nuevas disposiciones apuntando, pienso sin querer, a aumentar las desigualdades en la sociedad. Se invitó a reforzar la vacunación con más dosis de las pensadas originalmente, por la nueva evidencia que aparecía indicando esta nueva necesidad, sin enfatizar lo suficiente en los no vacunados. De acuerdo con los datos periódicos de la Encuesta de Pulso Social del DANE ${ }^{16}$, las personas no vacunadas están así principalmente por no tener tiempo para ir a vacunarse. Esto no debería entenderse de manera literal, sino hacer una lectura profunda que quizá indique al mismo tiempo una baja percepción de riesgo, como dificultades reales para acceder a las vacunas. De acuerdo con datos de diciembre de 2021, la vacunación más exitosa estaba ligada a las grandes ciudades de las regiones montañosas; en zonas dispersas, rurales, donde habita gran parte de la población más vulnerable los rezagos eran enormes. Aquí es muy importante resaltar que desde una perspectiva poblacional vacunar a los individuos con más 
riesgo (más edad y con multimorbilidad) ${ }^{17}$ y la primera dosis de la vacuna son lo más lo más importante; después ya viene en importancia la segunda dosis y luego el refuerzo.

De allí que privilegiar los refuerzos es una política fuertemente medicalizada, que enfatiza en la protección individual por encima de la poblacional. ¡Y qué pensar de que en enero de 2022 ya se empieza a hablar de una cuarta dosis, segunda de refuerzo! Como se puede ver, las intervenciones poblacionales de vigilancia en salud pública han perdido importancia en el manejo de la pandemia, y la vacunación infantil ${ }^{17}$ y los refuerzos vacunales han sido las intervenciones más favorecidas, evidencia de la medicalización del manejo de la pandemia... ¡la salud pública vuelve a perder!

\section{Referencias}

1. Nye RA. The evolution of the concept of medicalization in the late twentieth century. J Hist Behav Sci 2003; 39(2): 115-129. doi: https://doi.org/10.1002/jhbs.10108

2. Van Dijk W, Meinders MJ, Tanke MAC, Westert GP, Jeurissen PPT. Medicalization defined in empirical contexts - A scoping review. Int J Health Policy Manag 2020; 9(8): 327-334. doi:10.15171/ijhpm.2019.101

3. Conrad P. The shifting engines of medicalization. J Health Soc Behav 2005; 46(1): 3-14. doi: https://doi. org/10.1177/002214650504600102

4. Rose G. The strategy of preventive medicine. Oxford: Oxford University Press; 1992.

5. Rose G. Sick individuals and sick populations. Int J Epidemiol 1985; 14(1): 32-38. doi: https://doi.org/10.1093/ ije/30.3.427

6. McKeown T. The role of medicine: dream, mirage, or Nemesis? London: Nuffield Provincial Hospitals Trust; 1976.

7. Bunker JP. The role of medical care in contributing to health improvements within societies. Int J Epidemiol 2001; 30(6): 1260-1263. doi: https://doi.org/10.1093/ije/30.6.1260

8. Kaplan RM, Milstein A. Contributions of health care to longevity: a review of 4 estimation methods. Ann Fam Med 2019; 7(3): 267-272. doi: https://doi.org/10.1370/afm.2362

9. Buxbaum JD, Chernew ME, Fendrick AM, Cutler DM. Contributions of public health, pharmaceuticals, and other medical care to US life expectancy changes, 1990-2015. Health Aff (Millwood) 2020; 39(9): 1546-1556. doi: https://doi.org/10.1377/hlthaff.2020.00284

10. Hurtado-Ortiz A, Moreno-Montoya J, Prieto-Alvarado FE, Idrovo AJ. Evaluación comparativa de la vigilancia en salud pública de COVID-19 en Colombia: primer semestre. Biomédica 2020; 40: 96-103.

11. Idrovo AJ, Moreno-Montoya J. Diagnóstico con RT-qPCR en laboratorios departamentales de salud pública como determinantes del desempeño de la vigilancia en salud pública durante la pandemia. Biomédica. 2021; 41(2): 378-379.

12. Fernández-Niño JA, Peña-Maldonado C, Rojas-Botero M, Rodriguez-Villamizar LA. Effectiveness of contact tracing to reduce fatality from COVID-19: preliminary evidence from Colombia. Public Health. 2021; 198: 123128. doi: https://doi.org/10.1016/j.puhe.2021.07.013

13. Guzmán Ruiz Y, Vecino-Ortiz AI, Guzman-Tordecilla N, Peñaloza-Quintero RE, Fernández-Niño JA, RojasBotero M, et al. Cost-effectiveness of the COVID-19 test, trace and isolate program in Colombia. Lancet Reg Health Am. 2022; 6:100109. doi: https://doi.org/10.1016/j.lana.2021.100109

14. Idrovo AJ. More social discontent than pandemic-related risk perception in Colombia. Lancet 2021; 398(10296): 211. doi: https://doi.org/10.1016/S0140-6736(21)01239-3

15. Shultz JM, Berg RC, Bernal Acevedo OA, Ocampo Cañas JA, Escobar VAP, et al. Complex correlates of Colombia's COVID-19 surge. Lancet Reg Health Am 2021; 3:100072. doi: https://doi.org/10.1016/j. lana.2021.100072

16. Resultados encuesta Pulso Social. 2020. https://www.dane.gov.co/index.php/estadisticas-por-tema/encuestapulso-social

17. Bautista LE, Rodríguez-Villamizar LA, Herrera Galindo VM, Martínez Vega RA, Sosa Ávila LM, Oróstegui Arenas M, et al. Salud UIS 53: e21038. doi: https://doi.org/10.18273/saluduis.53.e:21038 